\title{
Cellular light memory, photo-electrochemical and redox retrograde signaling in plants
}

\author{
STANISEAW KARPIŃSKI ${ }^{1 *}$ AND MAGDALENA SZECHYŃSKA-HEBDA ${ }^{1,2}$ \\ ${ }^{1}$ Department of Plant Genetics, Breeding and Biotechnology, Faculty of Horticulture and Landscape Architecture \\ Warsaw University of Life Sciences, Warszawa, Poland \\ ${ }^{2}$ Institute of Plant Physiology, Polish Academy of Sciences, Kraków, Poland \\ *Corresponding author: stanislaw_karpinski@sggw.pl
}

\begin{abstract}
Plant chloroplasts emit signals that regulate the expression of nuclear-encoded genes, a process known as retrograde signaling. Environmental stresses, such as rapid and dynamic changes in light intensity and quality, temperature, relative humidity, water and $\mathrm{CO}_{2}$ availability, cause excess absorption of light energy (EEE) and induce chlorophyll fluorescence and heat dissipation, which lead to the generation of singlet stages of dioxygen, chlorophyll and carotenoid molecules. These primary quantum events in photosynthesis induce secondary redox reactions in photosystems, e.g. electrical charge separation, chloroplast lumen acidification and activation of the xanthophyll cycle by means of non-photochemical quenching (NPQ), redox reactions between the photosynthetic electron carriers (electron transport), and formation of reactive oxygen species. These, in turn, induce cascades of physiologically regulated redox reactions in the chloroplast stroma metabolism that regulate cellular light memory. Recently published data suggest that plants, with the help of EEE, NPQ, photoelectrochemical-redox retrograde signaling and cellular light memory, are able to perform biological processing in order to optimize their photosynthesis, transpiration, light acclimatory and defense responses, and in consequence, their Darwinian fitness. Understanding of the above-mentioned processes is crucial for future biotechnological amelioration of crop production.
\end{abstract}

Key words: cellular light memory, non-photochemical quenching, quantum-redox reactions, photosynthetic electron transport, reactive oxygen species, retrograde photo-electrochemical and hormonal signaling

\section{Introduction}

Oxygenic photosynthesis has created an oxygen-rich atmosphere and allowed for the evolution of a rich variety of heterotrophic life forms on Earth. Photosynthesis in plants is performed by specialized semiautonomous organelles called plastids, essential to almost all aspects of a plant's life. It is widely accepted that plastids originated from the engulfment of a photosynthetic bacterium by a eukaryotic cell circa 1.2 billion years ago (Douzery et al., 2004). The post-endo-symbiotic evolution of modern plant chloroplasts is characterized by a massive transfer of bacterial genes to the nucleus. In fact, approximately 2500 genes in the Arabidopsis genome that are required to build up a fully functional chloroplast, are now encoded in the nucleus, translated in the cytosol, and post-translationally imported into the organelle (Bedard and Jarvis, 2005). However, the remainder are encoded and synthesized within the organelle itself by its genetic system (Jarvis, 2007; Rogalski et al., $2008 \mathrm{a}, 2008 \mathrm{~b})$. Location of the genes encoding the organelle proteins in the different cellular compartments (nucleus and plastids) implies the existence of specific molecular and physiological mechanisms that orchestrate the coordination of nuclear and plastid gene expression to maintain plastid and cellular functions under different developmental stages and environmental conditions.

Cross-talk between organelles includes both anterograde (nucleus-to-organelle) and retrograde (organelle-tonucleus) signaling. In an anterograde regulation, nuclearencoded regulators that convey information on the cell type are able to regulate the expression of nuclear-encoded and plastid-encoded genes so that the proper stoichiometry of subunits of the plastid protein complexes is achieved for adequate development and functioning within a particular cell type (Pesaresi et al., 2007, 2009; Kleine et al., 2009; Jung and Chory, 2010). This type of 
regulation includes, inter alia, regulation of plastid development and activation of the plastid-encoded gene expression system (Chatterjee et al., 1996). In retrograde signaling, the expression of nuclear-encoded genes is regulated by plastid-specific signals. These signals reflect both the developmental and functional state of plastids. In the case of the absence of functional chloroplast development, light-stimulated expression of nucleus-encoded genes such as the light-harvesting chlorophyll $\mathrm{a} / \mathrm{b}$ binding protein (LHC) and Rubisco small subunit (RbcS) is repressed (Susek et al., 1993; Fankhauser and Chory, 1997; Mochizuki et al., 2008; Jung and Chory, 2010).

Once a seedling is established, most of the retrograde signals are dependent on the availability or limitation of light energy. Under conditions where the light energy absorbed is less than, or equal to, that required to support $\mathrm{CO}_{2}$ assimilation, the plant uses different redox signals to regulate its photosynthetic gene expression, compared to conditions when photosynthetically active photon flux density is in excess. Under lower light levels (up to $100 \mu \mathrm{mol}$ photons $\mathrm{m}^{-2} \mathrm{~s}^{-1}$ ), changes in light quality are relayed via signals that are generally believed to originate in or near the PQ pool (Asada, 2000). At higher irradiances $\left(300-1200 \mu \mathrm{mol}\right.$ photons $\left.\mathrm{m}^{-2} \mathrm{~s}^{-1}\right)$, redox signals are conveyed via the glutathione redox cycle and reactive oxygen species (ROS). When the absorption of light energy is in excess (EEE; 900-2500 $\mu \mathrm{mol}$ photons $\mathrm{m}^{-2} \mathrm{~s}^{-1}$ ) of that actually required for $\mathrm{CO}_{2}$ assimilation, the intersystem electron carriers are transiently over-reduced, which results in ROS-mediated damage to D1 protein and subsequent photoinhibition. Failure to dissipate EEE can be highly damaging to a plant's chloroplasts and often manifests itself as chlorosis, bleaching or bronzing of leaves due to the imbalanced metabolism of ROS (Karpinski et al., 1999; Niyogi, 2000; Apel and Hirt, 2004). However, in the recent decade it has been demonstrated that EEE induced ROS-signaling by abiotic stresses are perceived in the plastid (e.g. rapid increases in light intensity or temperature, or a reduction in relative humidity, water and $\mathrm{CO}_{2}$ availability) lead to effective plastid retrograde signaling. This type of signaling is essential for developmental and physiological processes, such as control of stomatal aperture to adjust optimal gas exchange, photosynthesis and transpiration, regulation of systemic acquired acclimation (SAA) and systemic acquired resistance (SAR) that are associated with programmed cell death (PCD) (Kwak et al., 2003; Melotto et al., 2006; Rossel et al., 2007; Muhlenbock et al., 2007, 2008; Szechyńska-Hebda et al., 2010). These pathways concern both intra- and extracellular retrograde signaling.

In the literature, there are limited data regarding the molecular basis of the mechanisms that coordinate the variety of retrograde signaling pathways and how, when and where these pathways interact. Due to their complexity, it is very difficult to separate their basic elements. Recently, we have demonstrated that an overexcited photosystem II (PSII) generates light wavelength-specific photo-electrochemical signaling that simultaneously regulates local and systemic cellular light acclimation and defense responses (Szechyńska-Hebda et al., 2010; Karpiński and Szechyńska-Hebda, 2010). We called this simultaneous induction of light acclimatory and defense responses a cellular light (quantum) memory because one single incident (e.g. $1 \mathrm{~h}$ ) of an excess white, red $(650 \mathrm{~nm})$ or blue $(450 \mathrm{~nm})$ wavelength of light is physiologically and specifically memorized for several days or longer (Szechyńska-Hebda et al., 2010). The electrochemical signals induced in response to different light treatments are also specific (Fig. 2A) and can be transduced from chloroplast to the nucleus, from chloroplasts to the plasma membrane by the chloroplast stromules and further by the membrane network in bundle sheath cells, and therefore, might be crucial in retrograde signaling (Fig. 2B). In this paper, we discuss the current knowledge and understanding of the role of photoelectrochemical signaling and redox reactions in retrograde signaling which is induced by EEE. Moreover, we attempt to answer the following question: How can the signal be transmitted within the cells, and communicated to the different tissues and organs and memorized?

\section{Pauli exclusion, principle and quantum-redox re- actions in plants}

Organisms in an oxygen-rich atmosphere have to deal with the dangers of oxidative stress. Plants especially are exposed to oxidative stress caused by photosynthetic processes. In a simplified model of photosynthesis, the absorbed photon energy can be used for photochemical reactions, induction of chlorophyll fluorescence and heat generation. When photosynthetically active photon flux density is in excess, the intersystem electron carriers are over-reduced and the larger part of the photon energy absorbed by the chlorophyll mole- 
cules, in the form of electron resonance, is transferred to oxygen molecules and results in the formation of ROS, such as: singlet oxygen $\left({ }^{1} \mathrm{O}_{2}\right)$, superoxide anion $\left(\mathrm{O}_{2}{ }^{--}\right)$, hydrogen peroxide $\left(\mathrm{H}_{2} \mathrm{O}_{2}\right)$ and other peroxides (Asada, 1999). The production rates of $\mathrm{O}_{2}{ }^{--}$and its dismutation product, $\mathrm{H}_{2} \mathrm{O}_{2}$, in one chloroplast of higher plants during photosynthesis are estimated at 240 and $120 \mu \mathrm{M} \mathrm{s}^{-1}$, respectively (Asada 1999). These amounts can inhibit Calvin cycle enzymes within seconds. Therefore, the existence of ROS scavenging systems, such as the waterwater cycle, are fundamental to maintaining photosynthetic activity and preventing the formation of the highly reactive and dangerous hydroxyl radical $\left({ }^{\circ} \mathrm{OH}\right)$ resulting from Fenton type reactions.

Oxygen in the ground (triplet) state $\left({ }^{3} \mathrm{O}_{2}\right)$ is a molecule with two unpaired electrons, each located in different $\pi^{*} 2 p$ antibonding orbitals. These two electrons have the same spin quantum number. Such an organization of electrons is energetically very unfavourable and naturally qualifies ${ }^{3} \mathrm{O}_{2}$ as a potential radical. One expression of the Pauli's exclusion principle is that no two electrons in the same atom can be in the same quantum state at the same time, therefore, addition of a pair of electrons to ${ }^{3} \mathrm{O}_{2}$ is forbidden. According to the Pauli's exclusion principle (a basic quantum physics law), oxygen activation may occur via two different mechanisms, e.g. monovalent reduction or absorption of sufficient energy to reverse the spin on one of the unpaired electrons. The monovalent reduction of the oxygen particle leads to the formation of superoxide $\left(\mathrm{O}_{2}{ }^{-}\right)$and hydrogen peroxide $\left(\mathrm{H}_{2} \mathrm{O}_{2}\right)$. However, if these two ROS were not separated in the sub-cellular space and in the presence of, e.g. $\mathrm{Fe}^{3+},{ }^{-} \mathrm{OH}$ could be formed and induce a chain reaction of, e.g. lipid peroxidation (Fridovich, 1997; Kobzev and Urvaev, 2006).

The major mechanism of singlet oxygen $\left({ }^{1} \mathrm{O}_{2}\right)$ production/scavenging in biological systems is dependent on the energy transfer between photo-excited pigments. In photosynthetic organisms, ${ }^{1} \mathrm{O}_{2}$ is primarily generated in photodynamic reactions between excited chlorophyll and ${ }^{3} \mathrm{O}_{2}$. Light absorption results in singlet-state excitation of a Chl a molecule $\left({ }^{1} \mathrm{Chl}^{*}\right)$. When this occurs, an alternative reaction can convert the singlet excited state into the triplet excited state $\left({ }^{3} \mathrm{Chl}^{*}\right)$. Therefore, the triplet pathway can be a significant valve for excess excitation (4-25\% of absorbed photons; Foyer and Harbinson, 1999), however, the energy of the triplet-excited chloro- phyll state $\left({ }^{3} \mathrm{Chl}^{*}\right)$ can simultaneously be transferred directly to the other triplet molecules, since the reaction between the triplet molecules is not spin-forbidden. ${ }^{3} \mathrm{Chl}^{*}$ reacts with the ever present oxygen $\left(\mathrm{O}_{2}\right)$ to form ${ }^{1} \mathrm{O}_{2}$.

In terms of their antioxidant properties, carotenoids can protect the photosystems in several ways: by reacting with lipid peroxidation products to terminate chain reactions; by scavenging ${ }^{1} \mathrm{O}_{2}$ and dissipating the energy as heat; by the dissipation of EEE through the xanthophyll cycle; or by reacting with triplet or excited chlorophyll molecules to prevent the formation of ${ }^{1} \mathrm{O}_{2}$. In the PSII reaction center (P680), two molecules of $\beta$-carotene participate in the quenching of ${ }^{1} \mathrm{O}_{2}$ generated via ${ }^{3} \mathrm{P} 680^{*}$. However, the distance between $\beta$-carotene and P680 in PSII does not allow direct quenching of ${ }^{3} \mathrm{P} 680^{*}$ by $\beta$-carotene. Oxidation of $\beta$-carotene in $\mathrm{D}_{2}$ to its action radical has been demonstrated, indicating that this $\beta$-carotene participates in the cyclic electron flow with Cyt $b-559$ in PSII. This cyclic electron transfer through PSII would suppress the generation of ${ }^{3} \mathrm{P} 680$ * and then ${ }^{1} \mathrm{O}_{2}$ (Asada, 2006).

Taking into consideration the above-mentioned quantum-physics features of oxygen, its high concentration around photosystems (21\%), physical and chemical features of ROS and functional organization of pigments in light harvesting complexes (LHC) of photosystems, it is clear that ROS are "naturally born" messenger molecules in retrograde signaling. Therefore, retrograde signaling strength and specificity are directly dependent on photosynthetically active light intensity and its energy absorbed, on the spectral quality of light, and on the primary and secondary quantum reactions (Szechyńska-Hebda et al., 2010; Karpinski and Szechyńska-Hebda, 2010).

\section{Light-induced redox retrograde intra-cellular signa- ling}

In the classical view, EEE can be highly damaging to plants due to an imbalanced ROS metabolism (Asada, 1999; Karpinski et al., 1999; Karpinska et al., 2000; Niyogi, 2000; Apel and Hirt, 2004). However, the natural capacity to absorb light energy in excess evolved in plants and in the last decade, it was demonstrated that EEE-induced foliar chlorosis is, in fact, an induced PCD mechanism regulated by subsequent changes in the cellular ROS/hormonal/calcium cellular homeostasis, and not in changes in ROS alone (Mateo et al., 2004; Chang 


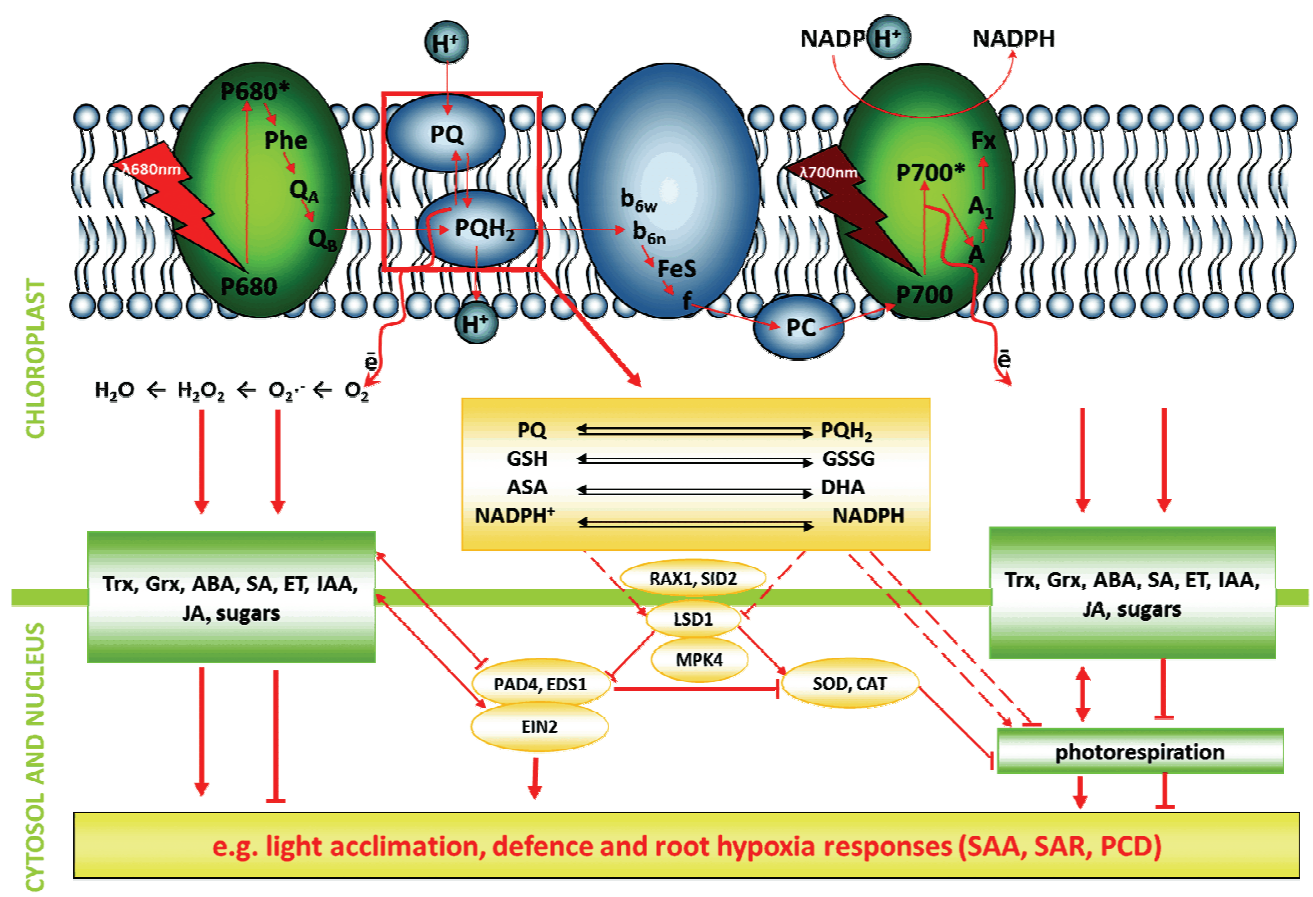

Fig. 1. Signals originating from chloroplasts that regulate nuclear gene expression. Primary and secondary changes in the quantumredox status of the photosynthetic electron transport components lead to the generation of ROS and changes in the redox status of the plastoquinone, glutathione, ascorbate and NADP pools, which in turn regulate retrograde signaling acclimatory and defense responses. Retrograde signaling is also regulated by the thioredoxin and glutaredoxin system. Moreover, post-translational modifications and metabolic pathways, e.g. hormone and sugar synthesis, have been reported in the regulation of retrograde signaling. Abbreviations: ABA - abscisic acid; ASA - ascorbate; CAT - catalase; DHA - dehydroascorbate; EDS1 - enhanced disease susceptibility; EIN2 - ethylene insensitive 2; ET - ethylene, GRX - glutaredoxin; GSH - reduced glutathione; GSSG - oxidized glutathione; IAA - indolilo-3acetic acid; LSD1 - lesion simulating disease 1; MPK4 - mitogen-activated protein kinase 4; PQ plastoquinone; PAD4 - phytoalexin deficient 4; PCD - programmed cell death; ROS - reactive oxygen species; SA - salicylic acid;

SAA - systemic acquired acclimation; SAR - systemic acquired resistance; SOD - superoxide dismutase; TRX - thioredoxin

et al., 2004; Baier and Dietz, 2005; Mühlenbock et al., 2007; 2008; Ślesak et al., 2007; Van Breusegem et al., 2008; Galvez-Valdivieso et al., 2009; Kleine et al., 2009; Szechyńska-Hebda et al., 2010).

Several types of retrograde intra-cellular signals can be distinguished (Fig. 1) due to the fact that the signals originate from primary (from rapidly changing light absorption and electron quantum numbers) or secondary quantum events (to the arrays of physiological photoelectrochemical and redox reactions). Since the reactions occurring upon the absorption of photon energy (primary quantum events) are several orders of magnitude faster than the redox reactions (secondary quantum events), the speed of the reactions on each subsequent level of complexity is substantially reduced (Fig. 1).

In type one retrograde signaling, the primary quantum events signals are derived from the excited singlet stages of photo-activated chlorophyll molecules in PSII
(Fig. 1). The absorption of the photon energy by the valence electron of the $\mathrm{Mg}$ atom in the chlorophyll molecule is followed by electron resonance within the LHCII, with simultaneous energy dependent quenching as a heat (induction of $\mathrm{NPQ}$, particularly its component $\mathrm{qE}$ which results in the generation of ${ }^{1} \mathrm{O}_{2}$, and ${ }^{1} \mathrm{Car}^{*}$ ) and fluorescence, and heat of the appropriate photon. Such primary quantum events are followed by secondary quantum events that cause acidification of the chloroplast lumen, activation of the xanthophyll-cycle (Krause et al., 1982; Yamamoto et al., 1999; Baker 2008) and activation of abscisic acid (ABA) synthesis and ABA/ROS signaling (Fryer et al., 2003; Apel and Hirt, 2004; Fey, 2004; Laloi et al., 2007; Galvez-Valdivieso and Mullineaux, 2010; Szechyńska-Hebda et al., 2010). It was demonstrated in field experiments that NPQ, (NPQ is a mechanism dependent upon lumen acidification) is a prerequisite for proper light acclimatory responses and the regulation of 
$A P X 1$ and $A P X 2$ gene expression (Kulhaim et al., 2002; Szechyńska-Hebda et al., 2010). In this work we demonstrate that in low light conditions (no EEE), $A P X 1$ and APX2 gene expression, robust SAA molecular markers, are induced in $n p q 4$ recessive mutant that has not functional $\mathrm{PsbS}$ protein. In addition to the documented regulatory role of $\mathrm{NPQ}$, changes in it may potentially cause fluctuations in the chloroplast volume due to heat dissipation. Cytosolic molecular temperature sensors and secondary messenger systems connected with them may activate specific transcription factors and transduce this type of information to the nucleus. However, according to our knowledge, no experimental evidence has been published that would prove the existence of this hypothetical component of retrograde signaling.

Type two signaling is several orders of magnitude slower (than type one) and is induced by secondary quantum events (photoelectrochemical and redox reactions) (e.g. Dirac, 1982) (Fig. 1 and Fig. 2). Signaling is triggered by changes in the physical electron transfer between the atoms of an electron valence orbital donors and acceptors and consists of the following: 1) redox changes in the photosynthetic electron carriers of PSII and in its proximity, e.g. the redox status of the plastoquinone pools $\left(\mathrm{PQ} / \mathrm{PQH}_{2}\right)$, glutathione $(\mathrm{GSH} / \mathrm{GSSG})$, ascorbate (ASA/DHA) (Pfannschmidt et al., 1999; Karpinska et al., 2000; Fey et al., 2005; Foyer and Noctor, 2005; Bräutigam et al., 2009; Pesaresi et al., 2009) and 2) redox signals generated downstream of PSI and linked: to the cyclic electron flow (Asada, 1999), to the redox status of NADP/NADPH, to the ferredoxin (Frx), thioredoxin (Trx) and glutaredoxin (Grx) activities (Pfannschmidt et al., 2001; Dietz et al., 2002; Fey, 2004; Baier et al., 2005; Rouhier et al., 2005; Mittler, 2006; Bräutigam et al., 2009; Bashandy et al., 2010; Arsova et al., 2010) (Fig. 1). In this type of retrograde signaling, the redox active molecules (e.g. glutathione or its precursor) can be exported from the chloroplast to the cytoplasm (Marty et al., 2009), and can regulate appropriate redox sensors.

Recently, we demonstrated that glutathione and salicylic acid (SA) retrograde signaling are genetically and physiologically interconnected and dependent on each other in the regulation of light acclimatory, defense and growth responses (Fig. 1), (Mateo et al., 2006; Mühlenbock et al., 2007, 2008). The molecular regulators of $\mathrm{Le}$ sion Simulating Disease 1 (LSD1), Enhanced Disease
Susceptibility 1 (EDS1) and Phytoalexin Defficient 4 (PAD4) were studied with regard to the cell death-dependent immune-defense response, root hypoxia and light acclimatory responses in Arabidopsis. LSD1, a negative regulator of EDS1 and PAD4, was mainly studied in the context of ROS- and SA-dependent response to pathogens (Jabs et al., 1996; Kliebenstein et al., 1999; Resterucci et al., 2001). Later, we demonstrated that apart from its role in pathogenesis, LSD1 is required for an efficient acclimation to growth conditions that promote EEE. The deprived ability of EEE dissipation, deregulated stomatal conductance and photorespiration underlie the cell death in the $1 s d 1$ mutant, which is dependent on EDS1 and PAD4 (Mateo et al., 2004; Mühlenbock et al., 2007, 2008). These and other studies suggest a general functional linkage between LSD1, EDS1 and PAD4 genes in the orchestrated regulation of retrograde signaling in simultaneous responses to biotic and abiotic stresses. Recently, we identified Trx binding domains within the LSD1 protein sequence. The transcription of many nuclear genes, including $L H C, R b c S$, ELIP, APX1, APX2 and PR1, is directly dependent on type two retrograde signaling. Therefore, the interaction between ROS generated by PSII and PSI, ASA and GSH, SA and NADP is crucial for the total sum of the retrograde signal (Mateo et al., 2006; Mühlenbock et al., 2007, 2008).

Glutathione reacts chemically with a range of ROS, while enzyme-catalysed reactions link GSH to the detoxification of $\mathrm{H}_{2} \mathrm{O}_{2}$ in the ascorbate-gluthatione cycle (Noctor et al., 2002). Moreover, the water-water cycle effectively shortens the lifetimes of photoproduced $\mathrm{O}_{2}{ }^{--}$and $\mathrm{H}_{2} \mathrm{O}_{2}$, and in consequence they reduce the probability of generating ${ }^{-} \mathrm{OH}$. These mechanisms, together with the down-regulation of PSII (by means of NPQ), allow to suppress ROS interactions with the target molecules in chloroplasts and safely dissipate EEE and excess of electrons, thus preventing photoinhibition (Asada, 1999). Plant cells are able to transport chloroplastically-produced gluthatione and both forms of glutathione (GSH and GSSG) can be transported, although GSH appears to be preferred (Foyer and Noctor, 2011). As a result, together with cytoplasmically-produced glutathione, they also protect proteins against oxidation and denaturation in the cytosol. The location of ascorbate peroxidase (APX) and related ascorbate-glutathione cycle enzymes indicates that chloroplasts also reduce $\mathrm{H}_{2} \mathrm{O}_{2}$ with the in- 
volvement of other antioxidative mechanisms by using electrons derived from water in PSII. Furthermore, this type of signaling is also regulated by ethylene (ET), salicylic acid (SA), jasmonic acid (JA) and auxin synthesis (IAA) (Wildermuth et al., 2001, Mateo et al., 2004, 2006; Mühlenbock et al., 2007, 2008; Bräutigam et al., 2009) which, in turn, regulate and are regulated in the forward signaling loop by the photorespiration process (Kozaki and Takeba, 1996; Mateo et al., 2004; Mateo et al., 2006; Mühlenbock et al., 2008) - Fig. 1.

Type one and type two retrograde signaling are strongly interconnected and influence each other. Many of these processes and interaction pathways, as well as their importance, are still the subject of debate among researchers (Karpinska et al., 2000; Mullineaux et al., 2000; Ślesak et al., 2003; Mateo et al., 2004; Ball et al., 2004; Mateo et al., 2006; Geisler et al., 2006; Mühlenbock et al., 2007, 2008; Yasuda et al., 2008; Bräutigam et al., 2009; Arsova et al., 2010; Bashandy et al., 2010; Szechyńska-Hebda et al., 2010; Foyer and Noctor, 2011; Foyer and Shigeoka, 2011).

Type three retrograde metabolic signaling includes end products, such as sugars and their derivatives, tocopherol synthesis, and chlorophyll anabolism and catabolism intermediates (Papenbrock et al., 2001; Pruzinska et al., 2003; Strand et al., 2003; Chang et al., 2004; Mateo et al., 2004; Geisler et al., 2006; Ankele et al., 2007). Geisler and colleagues (2006) demonstrated that ROS and sugar signaling are integrated by the presence of specific ROS/sugar cis-regulatory elements. They demonstrated that the identical cis-regulatory elements react upon changes of $\mathrm{H}_{2} \mathrm{O}_{2}$ and sugar concentration.

Type four, recently discovered photoelectrochemical signaling, is triggered by type one and type two retrograde signaling (Fig. 2A and, Fig. 2B) (Szechyńska-Hebda et al., 2010; Karpinski and Szechyńska-Hebda, 2010) and corresponds to the intracellular transitions of chloroplast-originated signals by the chloroplast stromules. Plastids are usually regarded as separate individual organelles within plant cells. However, there is now a body of evidence, accumulated over a period of one hundred years or so, which indicates that plastids are interconnected with other plastids, with the nucleus and plasma membrane by the stromules that form a cellular network of extended chloroplast envelope membranes (Senn, 1908; Kwok and Hanson, 2003a, 2003b; Hanson and Sattarzadeh, 2008). The stromules are enclosed by the inner and outer plastid envelope membranes and are $0.75 \mu \mathrm{m}$ in diameter and up to $6.5 \mu \mathrm{m}$ in length for Arabidopsis (Tirlapur et al., 1999). Therefore, stromule production provides a means for maximizing plastid surface area, which may be essential for optimizing metabolic, signal transduction and communication processes that require the exchange of macromolecules (substrates and products) or signals across the plastid envelope (e.g. the effective transition of photo-electrochemical and redox potential) - Fig. 2A and Fig. 2B (Köhler et al., 1997). It was also demonstrated that the stromule helps with the chloroplast movements. Interconnected chloroplasts are brought up along the cytoskeletal strands at speeds of about $1.5 \mu \mathrm{m} / \mathrm{s}$ (Menzel, 2001), and effectively deliver the signaling molecules to the target place in the cell.

Generally, the four listed types of retrograde signaling are interdependent, overlapping and interfering, simultaneously regulating light, temperature and humidity dependent developmental, acclimatory and defense responses in plants. These types of signaling are processed by the redox regulatory hubs, e.g. LSD1, EDS1 and PAD4, located in the cytosol and, when activated, act as transelements that are moved to the nucleus (Fig. 1), and conditionally regulate the expression of many genes. LSD1 is necessary for acclimation to conditions that promote EEE stress. LSD1 positively regulates, either directly or indirectly, e.g. superoxide dismutase (SOD) and catalase (CAT) gene expression and activities, and thus controls cellular ROS production (Jabs et al., 1996; Kliebenstein et al., 1999; Mateo et al., 2004). These regulatory traits depend on the LSD1 suppressive mode of the EDS1 and PAD4 function. LSD1/EDS1/PAD4 were shown to regulate optimal nuclear responses to inducing programmed cell death, light acclimation and defense responses (Mateo et al., 2004; Mühlenbock et al., 2007, 2008 ), and moreover, to influence the growth and healthy seed yield - major factors determining a plant's Darwinian fitness under natural conditions. It is also known that mitogen-activated protein kinases cascades are involved in SA signaling (Li et al., 2006). Total transcript profiling of a recessive mpk4 mutant identified that many nuclear-encoded chloroplast proteins, including PsbS, were deregulated in this mutant (Petersen et al., 2000).

We conclude that due to the complexity of the signaling pathways, experiments that argue for specific signa ling are partial and may be seriously misleading for 


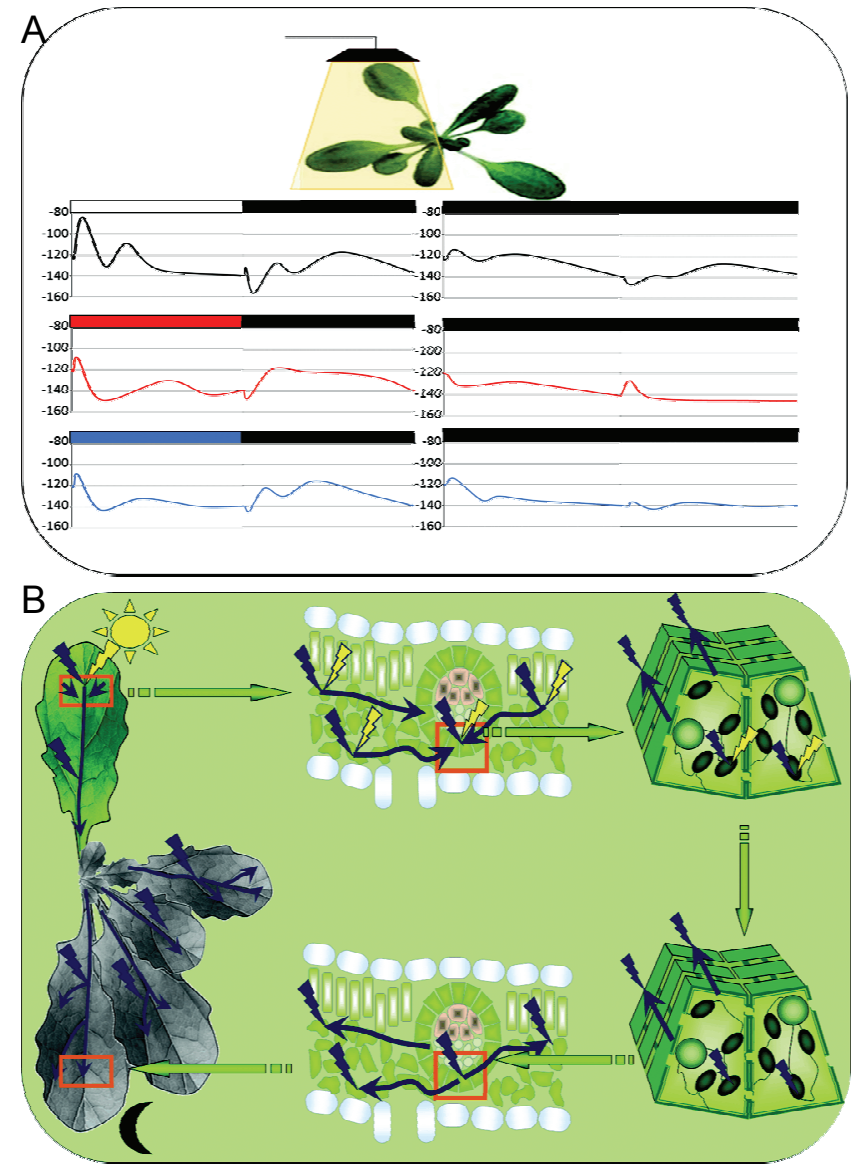

Fig. 2. Systemic acquired acclimation is induced by wavelength-specific photo-electrochemical signaling. (A) Arabidopsis Col-O rosettes grown under low-light (LL; $100 \mu \mathrm{mol}$ photons $\mathrm{m}^{-2} \mathrm{~s}^{-1}$ ) conditions were partially exposed to excess light (1500 $\mu \mathrm{mol}$ photons $\left.\mathrm{m}^{-2} \mathrm{~s}^{-1}\right)$. The light was directed onto a single leaf by an optical fiber and selective filters of 650 (red) and 450-(blue) nm wavelength were used when required (Szechyńska-Hebda et al., 2010; Karpiński and SzechyńskaHebda, 2010). Changes in the plasma membrane electrical potential of the bundle sheath cells were measured by impaling a cell with a microelectrode. The tip of one microelectrode was inserted into the bundle sheath cell of the locally treated leaves, and the tip of a second electrode was placed in the bundle sheath cell of the systemic leaves. The systemic leaf of a partially exposed Arabidopsis rosette was in the twilight zone. The light was turned on and off during the experiment at 20 min intervals, and the action potential was measured simultaneously for a period of $40 \mathrm{~min}$ in two separated single bundle sheath cells (in local and systemic leaves) by a coupled system of Axoclamp-2B (Axon Instruments, CA, USA) as described in Szechyńska-Hebda et al., 2010. (B) A specific photo-electrochemical signal (blue lightning and arrows symbol), induced by light (yellow lightning symbol) is transduced, at least in part, by the bundle sheath cell layer to systemic leaves (non-treated with excess light). For the purpose of the photo-electrochemical signal transduction, plants can use chloroplast stromules and plasmodesmata that form a global network of chloroplasts the correct understanding and proper description of the specific role of a given gene or signaling molecule. Such a partial description could be observed in defining the role of, for example, SA in exclusive regulation of biotic responses or in specific signaling is induced by ${ }^{1} \mathrm{O}_{2}$. It has been demonstrated that role of $\mathrm{SA}$ in biotic stress responses is only ancillary, and it mainly regulates growth, photosynthesis and development under variable abiotic stress conditions (Mateo et al., 2004, 2006; Mühlenbock et al., 2008) (Figs. 1 and 3).

\section{Light-induced redox retrograde extra-cellular signa- ling}

Recent results and those published earlier indicate that communication between the chloroplasts of distant cells in different organs is possible at the whole-plant level (Karpinski et al., 1999; Fryer et al., 2003; Szechyńska-Hebda et al., 2010; Karpiński and Szechyńska-Hebda, 2010). Higher levels of ${ }^{1} \mathrm{O}_{2}$ and ${ }^{1} \mathrm{Chl}^{*}$ (the last one was detected by changes in NPQ) were generated in the Arabidopsis rosette partly exposed to excess light. NPQ changes occurred in both parts of the rosette, i.e. in leaves that were directly illuminated with excess light and in leaves of the rosette that were kept in the low light zone (shadow), in comparison to a low light grown rosette (Fig. 4). This indicates that systemic changes of NPQ are part of the extra-cellular retrograde signaling, which can induce SAA (Karpinski et al., 1999; Fryer et al., 2003; Szechyńska-Hebda et al., 2010; Karpiński and Szechyńska-Hebda, 2010). ${ }^{1} \mathrm{O}_{2}$ can diffuse at a short distance from its generation place; therefore, it is clear that singlet molecules cannot be considered as long-distance extra-cellular retrograde signaling messengers. Therefore, ${ }^{1} \mathrm{O}_{2}$ and ${ }^{1} \mathrm{Chl}^{*}$ and systemic changes in NPQ are generated systemically by another type of long-distance retrograde signaling distinguished between the chloroplasts of directly stressed cells and the chloroplasts of the remote cells. An appropriate candidate that is able to regulate systemic quantum-redox changes $(\mathrm{NPQ}$ and generation of ${ }^{1} \mathrm{O}_{2}$ ) in naive chloroplasts and PSII seems to be the recently discovered photo-electrochemical retrograde signaling mechanism (Szechyńska-Hebda et al., 2010; Karpiński and Szechyńska-Hebda, 2010) - Fig. 2 and Fig. 4. Such a mechanism comprises the extra-cellular transduction of signals that are transduced by the phloem and bundle sheath cell layer connecting different leaf parts 


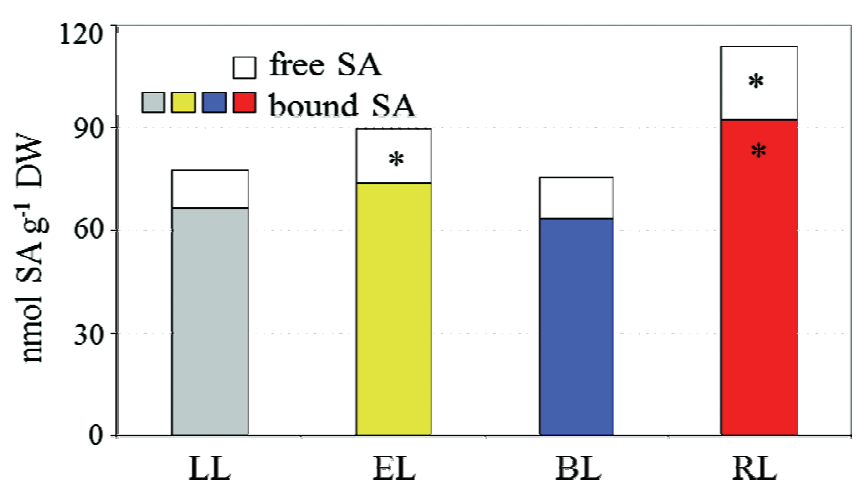

Fig. 3. Different light incidents differentially regulate foliar salicylic acid (SA) content. Arabidopsis leaves were exposed to excess light (EL, $1500 \mu \mathrm{mol}$ photons $\mathrm{m}^{-2} \mathrm{~s}^{-1} ; 60 \mathrm{~min}$ ), blue (BL; $80 \mu \mathrm{mol}$ photons $\mathrm{m}^{-2} \mathrm{~s}^{-1} ; 4 \mathrm{~h}$ ) and red light (RL; $120 \mu \mathrm{mol}$ photons $\mathrm{m}^{-2} \mathrm{~s}^{-1} ; 4 \mathrm{~h}$ ). Free and bound foliar SA content was determined immediately after light treatment as described in Mühlenbock and coworkers (2008). The results represent the average of $n=5$ replicates from one experiment. Data were tested for significance by $t$-test. The asterisk $\left(P \leq 0.05^{*}\right)$ indicated significant differences in comparison to the low light control

and different organs, e.g. excess light-treated leaf with leaves kept in the shadow (Fig. 2B and Fig. 4), (Szechyńska-Hebda et al., 2010). Changes in the electrochemical potential of the cellular plasma membrane are the most rapid physiological reactions known in plants (in the order of $\mu$ s to ms) (Wheeler and Brownlee, 2008). They are also well-documented regulators of different signaling cascades in animals and plants (Burdon-Sanderson, 1873; Darwin, 1875; Bose, 1926; Pickard, 1973; Wildon et al., 1992; Trewavas, 2003; Lautner et al., 2005). Selfpropagating changes in the plasma membrane electrochemical potential were described to be a systemic signaling component of the mechanism regulating SAA and SAR (Szechyńska-Hebda et al., 2010). Systemic changes in the electrochemical potential propagated via the bundle sheath cells is successful only in the case of intraand extra-cellular membrane integrity (stromule, plasmamembrane and their extension - plasmodesmata) and functional ion channels. If $\mathrm{LaCl}_{3}$ or DCMU (3-(3, 4-dichlorophenyl)-1,1-dimethylurea) treatment or mechanical damage was made to the petiole of a leaf directly exposed to excess light, then such a leaf was unable to communicate electrochemical signaling changes to the systemic leaves and to induce SAA and SAR (Mühlenbock et al., 2008; Szechynska-Hebda et al., 2010). We conclud that this extra-cellular retrograde signaling is governed by extra-cellular homeostasis of different hormones and ROS, and light specific changes in PSII quantum-redox properties of PSIIs experiencing EEE and concomitant changes in naive PSIIs that never experienced EEE. This hypothesis is strengthened by observations of the systemic wavy-like discrete in time and space changes of NPQ that correlates with antagonistic wavy-like discrete in time and space changes in $\mathrm{H}_{2} \mathrm{O}_{2}$, induction of $A P X 1$ and $A P X 2$ and stomatal conductance (Peak et al., 2004; Mühlenbock et al., 2007, 2008; Szechynska-Hebda et al., 2010).

The systemic discrete in time and space wavy pattern of changes in NPQ (Fig. 4) actually resulted from the appropriate pattern of prior changes in $\Delta \mathrm{pH}$ across the thylakoid membrane in remote cells and chloroplasts. This was demonstrated indirectly in our earlier experiments in which we proved that the PsbS dependent NPQ mechanism is a prerequisite for the regulation of SAA molecular markers (Szechynska-Hebda et al., 2010). This requires a very precise signaling mechanism that will regulate and coordinate which chloroplasts in which leaf sector should have a lower or higher $\mathrm{pH}$ value in the chloroplast lumen. It is also known that different $\mathrm{pH}$ values in the chloroplast lumen will differently change the redox status of the photosynthetic electron carriers, and therefore, they will differently regulate/deregulate retrograde signaling. To rephrase this information, we can say that different groups of chloroplasts and cells in the same leaf under identical stable light, temperature and relative humidity conditions have different "opinions as to what to do" (Fig. 4) and test different scenarios of possible future development (similar to different military operations during peacetime). However, the cells and leaves exposed to excess light represent one clear opinion: to use NPQ as efficiently as possible (Fig. 4) (like in a real war zone). Moreover, leaves that are still in the shadow also change NPQ in comparison to that before light treatment (just like mobilisation for war). Is this a sort of physiological exercise of naive (untreated) leaves?

\section{Cellular light memory, acclimation and plant "intel- ligence"}

It is always argued that although plants are capable of adaptation, this ability should not be called intelli gence. However, plant intelligence fits in with the definition of intelligence proposed by Stenhouse (1974) as "adaptively variable behavior during the lifetime of 


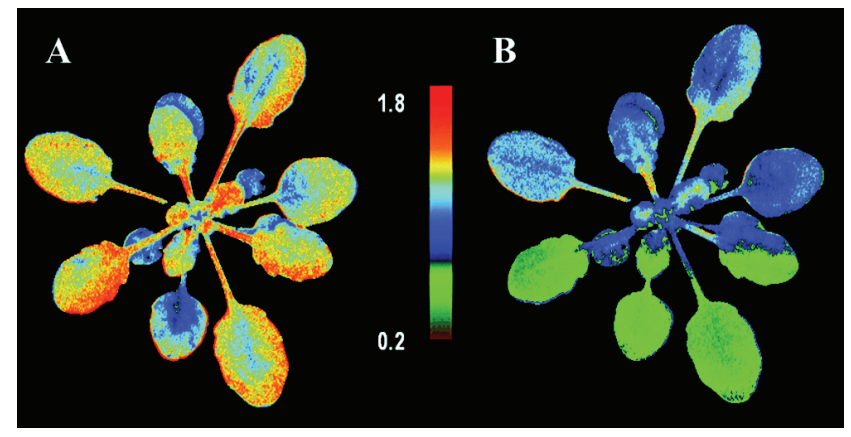

Fig. 4. Partial exposure to excess light and induction of SAAR is associated with systemic gradient-like changes of foliar NPQ and changes in PEPS. Arabidopsis thaliana Col-0 rosettes were grown at low-light conditions ( $\mathrm{LL}, 100 \mu \mathrm{mol}$ photons $\mathrm{m}^{-2} \mathrm{~s}^{-1}$ ) and were partially exposed to excess light (EL, 2,000 $\mu \mathrm{mol}$ photons $\mathrm{m}^{-2} \mathrm{~s}^{-1}$ ). (A) Left, controls that were LL grown with no excess light exposure. (B) Right, the same rosette partially exposed to EL for $60 \mathrm{~min}$ (three bottom leaves with the lowest NPQ value). NPQ estimated the nonphotochemical quenching from $F \mathrm{~m}$ to $F_{\mathrm{m}}^{\prime}$ is monitoring the apparent heat losses from PSII and is calculated from $\left(F_{\mathrm{m}} / F_{\mathrm{m}}^{\prime}\right)-1$, where $F_{\mathrm{m}}$ is maximal chlorophyll fluorescence of dark-adapted PSII (all QA molecules are reduced), $F_{\mathrm{m}}^{\prime}=$ is maximal chlorophyll fluorescence of light-adapted PSII(all QA molecules involved in photosynthetic electron transport from water in the provided light conditions are reduced)

the individual", and evaluated by Trewavas $(2003,2005)$ as "the ability of plants to sense the environment and adjust their morphology, physiology and phenotype accordingly". Studies indicate that plants are capable of problem-solving and communication, and can adapt their behavior (Farmer and Ryan, 2001). In plants, the mechanism responsible for adaptation is signal transduction, and reactions within the signaling pathways may provide a biochemical basis for learning and memory (an intelligent network like in Stanislaw Lem's "Solaris") (Bhalla and Iyengar, 1999). Moreover, it is known that plant leaves solve their problems of optimal gas exchange, transpiration and photosynthesis in a way that is similar to that defined by the algorithm of cellular automation (Peak et al., 2004; Mott and Peak, 2007). Cellular automation, as proposed by John von Neumann and Oskar Morgenstern (1944), is a mathematical model of a dynamic system, which is discrete in time and space. It depends on local interactions, but global phenomena emerge due to predefined local interactions. Basic elements of the cellular automata are a regular lattice of cells (e.g. in a leaf) in which at each time step each cell is in one defined "s" state. The cellular automata have a defined neighborhood behaving of cells in such a way that the state of each cell at time step " $t+1$ " is a function of some of its surrounding cells at time step " $t$ ". Peak and colleagues (2004) also demonstrated that dynamic and emergent changes in chlorophyll fluorescence are regulated by the cellular automation algorithm propagating across the leaf with the speed of circa $0.6 \mathrm{~cm}$ per second. The authors demonstrated that if a sinus function governs foliar fluorescence changes, then stomatal conductance is governed by 1 -sinus. However, they did not identify the hardware that determines such a pattern of changes. Our resent results described some parts of this hardware, which includes light-(quantum)-redox changes in PSII, its proximity (e.g. in NPQ, and the redox status of the glutathione and $\mathrm{PQ}$ pools), photoelectrochemical signaling, ROS/hormonal circuits, and finally cellular light memory, which determine the light acclimation and Darwinian fitness of a plant. Our results indicate that if a sinus function governs foliar NPQ changes, then ROS, ethylene and stomatal conductance changes are governed by 1-sinus (Mühlenbock et al., 2007, 2008; Szechynska-Hebda et al., 2010). We suggested that these antagonistic, emergent and discrete in time systemic foliar quantum-redox changes of NPQ, ROS, ethylene and stomatal conductance that are discrete in time and space are specifically regulated by the means of systemic photo-electrochemical retrograde signaling, induced by type one and type two intra-cellular retrograde signals. This photo-electrochemical retrograde signaling is propagated systemically with similar speed as the changes described by Peak and colleagues (2004) (Szechyńska-Hebda et al., 2010) (Fig. 4).

According to our knowledge, the mechanisms of training and memorizing in plants (acclamatory mechanisms) are not well known, and it is widely accepted that plants do not have such capacities (Trewavas, 2003). However, SAA is a training process of naive cells, chloroplasts and PSII (that have never experienced conditions that promote EEE), performed by cells, chloroplasts and PSII that are actually experiencing such conditions. As leaves are able to perform emergent, complex and distributed computation, as suggested previously (Peak et al., 2004), with the help of light-(quantum) memory and redox retrograde signaling, then plant cells are able to differentially memorize excess light incidents. Leaves in the dark are able to "see" the light (Karpinski et al., 1999; Foyer and Noctor, 1999), distinguish the spectral composition of light and differentially memorize episo- 
des of exposure to different absorbed photons energy (Szechyńska-Hebda et al., 2010). The cellular light memory of the excess light episode is maintained for at least several days. The plant cell is able to effectively use this memorized information just as the signaling pathways utilize a complex network of interactions to orchestrate biochemical and physiological responses to improve their light acclimation and chances of survival under prolonged periods of low light. This is probably the most elegant system that has evolved in complex photosynthetic organisms since it uses absorbed excess photon energy by some leaves to improve the survival chances of the whole plant. Animals have their network of neuron synapses, electrophysiological circuits and memory. Plants, however, have a chloroplast global network connected by stromules within mesophyll and bundle sheath cells, light-(quantum)-redox and electrochemical retrograde circuits and cellular light memory.

\section{Acknowledgements}

The authors are grateful to the Polish Science Foundation for support from the Welcome 2008/1 project operated within the Foundation for Polish Science Welcome Programme, co-financed by the European Regional Development Fund.

\section{References}

Ankele E., Kindgren P., Pesquet E., Strand A. (2007) In vivo visualization of Mg-protoporphyrin IX, a coordinator of photosynthetic gene expression in the nucleus and the chloroplast. Plant Cell 19; 1964-1979.

Apel K., Hirt H. (2004) Reactive oxygen species: metabolism, oxidative stress, and signal transduction. Ann. Rev. Plant Biol. 55: 373-399.

Arsova B., Hoja U., Wimmelbacher M., Greiner E., Ustun S., Melzer M., Petersen K., Lein W., Bornke F. (2010) Plastidial thioredoxin $z$ interacts with two fructokinase-like proteins in a thiol-dependent manner: Evidence for an essential role in chloroplast development in Arabidopsis and Nicotiana benthamiana. Plant Cell 22: 1498-1515.

Asada K. (2006) Production and scavenging of reactive oxygen species in chloroplasts and their functions. Plant Physiol. 141: 391-396.

Asada K. (1999) The water-water cycle in chloroplasts: scavenging of active oxygens and dissipation of excess photons. Annu. Rev. Plant Physiol. Plant Mol. Biol. 50: 601-639.

Asada K. (2000) The water-water cycle as alternative photon and electron sinks. Phil. Trans. Royal Soc., London, Series B - Biol. Sci. 355: 1419-1430.

Baier M., Dietz K.J. (2005) Chloroplasts as source and target of cellular redox regulation: a discussion on chloroplast redox signals in the context of plant physiology. J. Exper. Bot. 56: 1449-1462.

Baier M., Kandlbinder A., Golldack D., Dietz K.J. (2005) Oxidative stress perception, signaling and response. Plant Cell Environ. 28: 1012-1020.

Baker N.R. (2008) Chlorophyll fluorescence: a probe of photosynthesis in vivo. Annu. Rev. Plant Biol. 59: 89-113.

Ball L., Accotto G.P., Bechtold U., Creissen G., Funck D., Jimenez A., Kular B., Leyland N., Mejia-Carranza J., Reynolds H., Karpinski S., Mullineaux P.M. (2004) An Arabidopsis mutant with raised ASCORBATE PEROXIDASE 2 expression reveals glutathione as a direct modulator of stress responsive gene expression. Plant Cell 1: 2448-2462.

Bashandy T., Guilleminot J., Vernoux T., Caparros-uizc D., Ljung K., Meyer Y., Reichheld J.P. (2010) Interplay between the NADP-linked thioredoxin and glutathione systems in Arabidopsis auxin signaling. Plant Cell 22: 376-391.

Bedard, J., and Jarvis P. (2005) Recognition and envelope translocation of chloroplast preproteins. J. Exp. Bot. 56: 2287-2320.

Bhalla US., Iyengar R. (1999) Emergent properties of networks of biological signaling pathways. Science 283: 381387.

Bonardi V., Pesaresi P., Becker T., Schleiff E., Wagner R., Pfannschmidt T., Jahns P., Leister D. (2005) Photosystem II core phosphorylation and photosynthetic acclimation require two different protein kinases. Nature 437: 1179-1182.

Bose J.C. (1926) The Nervous Mechanism of Plants. Langmans, Green and Company, London.

Bräutigam K., Dietzel L., Kleine T., Ströher E., Wormuth D., Dietz K.J., Radke D., Wirtz M., Hell R., Dörmann P., NunesNesi A., Schauer N., Fernie A.R., Oliver S.N., Geigenberger P., Leister D., Pfannschmidt T. (2009) Dynamic plastid redox signals integrate gene expression and metabolism to induce distinct metabolic states in photosynthetic acclimation in Arabidopsis. Plant Cell 21: 2715-2732.

Burdon-Sanderson J. (1873) Note on the electrical phenomena which accompany irritation of the leaf of Dionea muscipula in the excited and unexcited states. Proc. R. Soc. Lond. 21: 491-496.

Chang C.C.C, Ball L., Fryer M.J., Baker N.R., Karpinski S., Mullineaux P.M. (2004) Induction of ASCORBATE PEROXIDASE 2 expression in wounded Arabidopsis leaves does not involve known wound signaling pathways but is associated with changes in photosynthesis. Plant J. 38: 499-511.

Chatterjee M., Sparvoli S., Edmunds C., Garosi P., Findlay K., Martin C. (1996) DAG, a gene required for chloroplast differentiation and palisade development in Antirrhinum majus. EMBO J. 15: 4194-4207.

Darwin C. (1875) Insectivorous Plants. John Murray, London. Depege N., Bellafiore S., Rochaix J.D. (2003) Role of chloroplast protein kinase Stt7 in LHCII phosphorylation and state transition in Chlamydomonas. Science 299: 1572-1575.

Dietz K.J., Link G., Pistorius E. K., Scheibe R. (2002) Redox regulation in oxygenic photosynthesis. Prog. Bot. 63: 207-245. 
Dirac P.A.M. (1982) Principles of quantum mechanics. Oxford University Press.

Douzery E.J.P., Snell E.A., Bapteste E., Delsuc F., Herve P. (2004) The timing of eukaryotic evolution: does a relaxed molecular clock reconcile proteins and fossils?Proc. Natl. Acad. Aci. USA 101: 15386-15391.

Fankhauser C., Chory J. (1997) Light control of plant development. Ann. Rev. Cell Develop. Biol. 13: 203-229.

Farmer E.E., Ryan C.A. (1990) Interplant communication: airborne methyl jasmonate induces synthesis of proteinase inhibitors in plant leaves. Proc. Natl. Acad. Sci. USA 87: 7713-7716.

Fey V., Wagner R., Brautigam K., Pfannschmidt T. (2005) Photosynthetic redox control of nuclear gene expression. J. Exp. Bot. 56: 1491-1498.

Fey V., Wagner R., Brautigam K., Wirtz M., Hell R., Dietzmann A., Leister D., Oelmuller R., Pfannschmidt T. (2004) Retrograde plastid redox signals in the expression of nuclear genes for chloroplast proteins of Arabidopsis thaliana. J. Biol. Chem. 280: 5318-5328.

Fischer B.B., Krieger-Liszkay A., Eggen R.L. (2004) Photosensitizers neutral red (type I) and rose bengal (type II) cause light-dependent toxicity in Chlamydomonas reinhardtii and induce the Gpxh gene via increased singlet oxygen formation. Environ. Sci. Technol. 38: 6307-6313.

Foyer C.H., Noctor G. (2011) Ascorbate and glutathione: the heart of the redox hub. Plant Physiol. 155: 2-18.

Foyer C.H., Shigeoka S. (2011) Understanding oxidative stress and antioxidant functions to enhance photosynthesis. Plant Physiol. 155: 93-100.

Foyer C.H., Noctor G. (1999) Leaves in the dark see the light. Science 284: 599-601.

Foyer C.H., Noctor G. (2005) Redox homeostasis and antioxidant signaling: a metabolic interface between stress perception and physiological responses. Plant Cell 17: 1866-1875.

Foyer C.H., Harbinson J. (1999) Relationships between antioxidant metabolism and carotenoids in the regulation of photosynthesis. In: The Photochemistry of Carotenoids, eds. Frank, H.A., A.J. Young, G. Britton, R.J. Cogdell, Kluwer Academic Publishers, The Netherlands, p. 305.

Fridovich I. (1997) Superoxide anion radical (Obardot 2), superoxide dismutases, and related matters. J. Biol. Chem. 272: 18515-18517.

Fryer M.J., Ball L., Oxborough K., Karpinski S., Mullineaux P.M., Baker N.R. (2003) Control of ascorbate peroxidase 2 expression by hydrogen peroxide and leaf water status during excess light stress reveals a functional organization of Arabidopsis leaves. Plant J. 33: 691-705.

Galvez-Valdivieso G. and Mullineaux P.M. (2010) The role of reactive oxygen species in signaling from chloroplasts to the nucleus. Physiol Plant 138: 430-439.

Galvez-Valdivieso G., Fryer M.J., Lawson T., Slattery K., Truman W., Smirnoff N., Asami T., Davies W.J., Jones A.M.J., Baker N.R., Mullineaux P.M., (2009) The high light response in Arabidopsis involves ABA signaling between vascular and bundle sheath cells. Plant Cell 21: 21432162.
Geisler M., Kleczkowski L.A. and Karpiński S. (2006) A universal algorithm for genome-wide in silicio identification of biologically significant gene promoter putative cis-regulatory-elements; identification of new elements for reactive oxygen species and sucrose signaling in Arabidopsis. Plant J. 45: 384-398.

Gray J.C., Sullivan J.A., Hibberd J.M., Hansen M.R. (2001) Stromules: mobile protrusions and interconnections between plastids. Plant Biol. (Stuttgart) 3: 223-233.

Hanson M.R., Sattarzadeh A. (2008) Dynamic morphology of plastids and stromules in angiosperm plants. Plant Cell Env. 31: 646-657.

Jabs T., Dietrich R.A., Dangl J.L. (1996) Initiation of runaway cell death in an Arabidopsis mutant by extracellular superoxide. Science 273: 1853-1856.

Jarvis P. (2007) Intracellular signaling: chloroplast backchat. Curr. Biol. 17(14): 552-555.

Jung H.S., Chory J. (2010) Signaling between chloroplasts and the nucleus: can a systems biology approach bring clarity to a complex and highly regulated pathway?Plant Physiol. 152: 453-459.

Karpinska B., Wingsle G., Karpinski S. (2000) Antagonistic effects of hydrogen peroxide and glutathione on acclimation to excess excitation energy in Arabidopsis. IUBMB Life 50: 21-26.

Karpiński S., Szechyńska-Hebda M. (2010) Secret life of plants: From memory to intelligence. Plant Behav. Signal. 5(11): 1391-1394.

Karpinski S., Escobar C., Karpinska B., Creissen G., Mullineaux P.M. (1997) Photosynthetic electron transport regulates the expression of cytosolic ascorbate peroxidase genes in excess light stress. Plant Cell 9: 627-640.

Karpinski S., Reynolds H., Karpinska B., Wingsle G., Creissen G., Mullineaux P. (1999) Systemic signaling and acclimation in response to excess excitation energy in Arabidopsis. Science 284: 654-657.

Kleine T., Maier U.G., Leister D. (2009) DNA transfer from organelles to the nucleus: the idiosyncratic genetics of endosymbiosis. Annu. Rev. Plant Biol. 60: 115-138.

Kliebenstein D.J., Dietrich R.A., Martin A.C., Last R.L., Dangl J.L. (1999) LSD1 regulates salicylic acid induction of copper zinc superoxide dismutase in Arabidopsis thaliana. Mol. Plant-Microbe Interact. 12: 1022-1026.

Kobzev G.I., Urvaev D.G. (2006) The nature of molecular oxygen binding and activation in $\mathrm{Mn}-\mathrm{O}_{2}$ complex. J. Struct. Chem. 47: 608-615.

Köhler R.H., Cao J., Zipfel W.R., Webb W.W., Hanson M.R. (1997) Exchange of protein molecules through connections between higher plant plastids. Science 276: 2039-2042.

Kozaki A., Takeba G. (1996) Photorespiration protects C3 plants from photooxidation. Nature 384: 557-560.

Krause G.H., Vernotte C., Briantais J.M. (1982) Photoinduced quenching of chlorophyll fluorescence in intact chloroplasts and algae. Resolution into two components. Biochim. Biophys. Acta 679: 116-124.

Kulheim C., Agren J., Jansson S. (2002) Rapid regulation of light harvesting and plant fitness in the field. Science 297: 91-93. 
Kwak J.M., Mori I.C., Pei Z.M., Leonhardt N., Torres M.A., Dangl J.L., Bloom R.E., Bodde S., Jones J.D.G., Schroeder J.I. (2003) NADPH oxidase AtrbohD and AtrbohF genes function in ROS-dependent ABA signaling in Arabidopsis. EMBO J. 22: 2623-2633.

Kwok E.Y., Hanson M.R. (2003a) Microfilaments and microtubules control the morphology and movement of non-green plastids and stromules in Nicotiana tabacum. Plant J. 35: 16-26.

Kwok E.Y., Hanson M.R. (2003b) Stromules and the dynamic nature of plastid morphology. J. Microsc. 214: 124-137.

Laloi C., Stachowiak M., Pers-Kamczyc E., Warzych E., Murgia I., Apel K. (2007) Cross-talk between singlet oxygenand hydrogen peroxide-dependent signaling of stress responses in Arabidopsis thaliana. Proc. Natl. Acad. Sci. USA 104: 672-677.

Lautner S., Grams T.E.E., Matyssek R., Fromm J. (2005) Characteristics of electrical signals in poplar and responses in photosynthesis. Plant Physiol. 138: 2200-2209.

Leisinger U., Rufenacht K., Fischer B., Pesaro M., Spengler A., Zehnder A.J., Eggen R.I. (2001) The glutathione peroxidase homologous gene from Chlamydomonas reinhardtii is transcriptionally up-regulated by singlet oxygen. Plant Mol. Biol. 46: 395-408.

Li Q., Xie Q.G., Smith-Becker J., Navarre D.A., Kaloshian I. (2006) Mi-1-Mediated aphid resistance involves salicylic acid and mitogen-activated protein kinase signaling cascades. Mol. Plant Microbe Interact. 19: 655-664.

Marty L., Sialab W., Schwarzlanderc M., Frickerc M.D., Wirtza M., Sweetlovec L.J., Meyerb Y., Meyera A.J., Reichheldb J.P., Hella Rudiger (2009) The NADPH-dependent thioredoxin system constitutes a functional backup for cytosolic glutathione reductase in Arabidopsis. PNAS 106: 9109-9114.

Mateo A., Funck D., Muhlenbock P., Kular B., Mullineaux P.M., Karpinski S. (2006) Controlled levels of salicylic acid are required for optimal photosynthesis and redox homeostasis. J. Exp. Bot. 57: 1795-1807.

Mateo A., Mühlenbock P., Rustérucci C., Chi-Chen C., Miszalski Z., Karpinska B., Parker J.E., Mullineaux P.M., Karpinski S. (2004) The Lesion Simulating Disease (LSD1) gene is required for acclimation to conditions that promote excess excitation energy. Plant Physiol. 136: 28182830.

Melotto M., Underwood W., Koczan J., Nomura K., He S.Y. (2006) Plant stomata function in innate immunity against bacterial invasion. Cell 126: 969-980.

Mittler R. (2006) Abiotic stress, the field environment and stress combination. Trends Plant Sci. 11: 15-19.

Mochizuki N., Tanaka R., Tanaka T., Masuda T., Nagatani A. (2008) The steady-state level of Mg-protoporphyrin $I X$ is not a determinant of plastid-to-nucleus signaling in Arabidopsis. Proc. Natl Acad. Sci. USA 105: 15184-15189.

Mott K.A., Peak D. (2007) Stomatal patchiness and task-performing networks. Ann. Bot. 99: 219-226.

Mühlenbock P., Plaszczyca M., Mellerowicz E., Karpinski S. (2007) Lysigenous aerenchyma formation in Arabidopsis is controlled by Lesion Simulating Disease1. Plant Cell 19: 3819-3830.

Mühlenbock P., Szechyńska-Hebda M., Płaszczyca M., Baudo M., Mullineaux P.M., Parker J.E., Karpińska B., Karpiński S. (2008) Chloroplast signaling and Lesion Simulating Disease 1 regulate crosstalk between light acclimation and immunity in Arabidopsis. Plant Cell 20: 2339-2356.

Mullineaux P., Ball L., Escobar C., Karpinska B., Creissen G., Karpinski S. (2000) Are diverse signaling pathways integrated in the regulation of Arabidopsis antioxidant defense gene expression in response to excess excitation energy? Philos. Trans. R. Soc. Lond. B Biol. Sci. 29; 355(1402): 1531-1540.

Niyogi K.K. (2000) Safety valves for photosynthesis. Curr. Opin. Plant. Biol. 3: 455-460.

op den Camp R.G., Przybyla D., Ochsenbein C., Laloi C., Kim C. Danon A., Wagner D., Hideg É., Göbel C., Feussner I., Nater M., Apel K. (2003) Rapid induction of distinct stress responses after the release of singlet oxygen in Arabidopsis. Plant Cell 15: 2320-2332.

Papenbrock J., Mishra S., Mock H.P., Kruse E., Schmidt E.K., Petersmann A., Braun H.P., Grimm B. (2001) Expression of ferrochelatase $m R N A$ in antisense orientation leads to a necrotic phenotype of transformed tobacco plants. Plant J. 28: 41-50.

Peak D., West J.D., Messinger S.M., Mott K.A. (2004) $E_{V i-}$ dence for complex, collective dynamics and emergent, distributed computation in plants. PNAS 101: 918-922.

Pesaresi P., Hertle A., Pribil M., Kleine T., Wagner R., Strissel H., Ihnatowicz A., Bonardi V., Scharfenberg M., Schneider A., Pfannschmidt T., Leister D. (2009) Arabidopsis STN7 kinase provides a link between short-and long-term photosynthetic acclimation. Plant Cell 21: 2402-2423.

Pesaresi P., Schneider A., Kleine T., Leister D. (2007) Interorganellar communication. Curr. Opin. Plant Biol. 10: 600606.

Petersen M., Brodersen P., Naested H., Andreasson E., Lindhart U., Johansen B., Nielsen H.B., Lacy M., Austin M.J., Parker J.E., Sharma S.B., Klessig D.F., Martienssen R., Mattsson O., Jensen A.B., Mundy J. (2000) Arabidopsis MAP kinase 4 negatively regulates systemic acquired resistance. Cell 103: 1111-1120.

Pfannschmidt T., Allen J. F., Oelmuller R. (2001) Principles of redox control in photosynthesis gene expression. Physiol. Plant 112: 1-9.

Pfannschmidt T., Nilsson A., Allen J. (1999) Photosynthetic control of chloroplast gene expression. Nature 397: 625628.

Pickard B.G. (1973) Action potentials in higher plants. Bot. Rev. 39: 172-201.

Rodermel S. (2001) Pathways of plastid-to-nucleus signaling. Trends Plant Sci. 10: 471-478.

Rogalski M., Karcher D., Bock R. (2008a) Superwobbling facilitates translation with reduced $t R N A$ sets. Nat. Struct. Mol. Biol. 15: 192-198.

Rogalski M., Schöttler M.A., Thiele W., Schulze W.X., Bock R. (2008b) Rpl33, a nonessential plastid-encoded ribosomal 
protein in tobacco, is required under cold stress conditions. Plant Cell 20: 2221-2237.

Rossel J.B., Wilson P.B., Hussain D., Woo N.S., Gordon M.J., Mewett O.P., Howell K.A., Whelan J., Kazan K., Pogson B.J. (2007) Systemic and intracellular response to photooxidative stress in Arabidopsis. Plant Cell 19: 40914110.

Rouhier N., Villarejo A., Srivastava M., Gelhaye E., Keech O., Droux M., Finkemeier I., Samuelsson G., Dietz K.J., Jacquot J.P., Wingsle G. (2005) Identification of plant glutaredoxin targets. Antioxid. Redox Signal. 7(7-8): 919929.

Rustérucci C., Aviv D.H., Holt III B.F., Dangl J.L., Parker J.E. (2001) The disease resistance signaling components EDS1 and PAD4 are essential regulators of the cell death pathway controlled by LSD1 in Arabidopsis. Plant Cell. 13: 2211-2224.

Senn G. (1908) Die gestalts- und lageveränderung der pflanzen-chromatophoren. Engelmann, Leipzig.

Ślesak I., Karpinska B., Surówka E., Miszalski Z., Karpinski S. (2003) Redox changes in the chloroplast and hydrogen peroxide are essential for regulation of C3-CAM transition and photooxidative stress responses in the facultative CAM plant Mesembryanthemum crystallinum L. Plant Cell Physiol. 44: 573-581.

Ślesak I., Libik M., Karpinska B., Karpinski S., Miszalski Z. (2007) The role of hydrogen peroxide in regulation of plant metabolism and cellular signaling in response to environmental stresses. Acta Biochim. Pol. 54: 39-50.

Stenhouse D. (1974) The evolution of intelligence - a general theory and some of its implications. George Allen and Unwin.

Strand A., Asami T., Alonso J., Ecker J.R., Chory J. (2003) Chloroplast to nucleus communication triggered by accumulation of Mg-protoporphyrinIX. Nature 421: 79-83.

Susek R.E., Ausubel F.M., Chory J. (1993) Signal transduction mutants of Arabidopsis uncouple nuclear $C A B$ and RBCS gene expression from chloroplast development. Cell 74: 787-799.

Szechyńska-Hebda M., Kruk J., Gorecka M., Karpinska B., Karpiński S. (2010) Evidence for light wavelength-specific systemic photoelectrophysiological signaling and cellular light memory of excess light episode in Arabidopsis. Plant Cell 22: 1-18.

Trewavas A. (2003) Aspects of plant intelligence. Ann. Bot. 92: $1-20$.

Trewavas A. (2005) Green plants as intelligent organisms. Trends Plant Sci. 10: 413-419.

Van Breusegem F., Bailey-Serres J., Mittler R. (2008) Unravelling the tapestry of networks involving reactive oxygen species in plants. Plant. Phys. 147: 978-984.

Villarejo A., Shutova T., Moskvin O., Forssen M., Klimov V.V., Samuelsson G. (2002) A photosystem II-associated carbonic anhydrase regulates the efficiency of photosynthetic oxygen evolution. EMBO J. 21: 1930-1938.

Von Neumann J., Morgenstern O. (1994) Theory of games and economic behavior. Princeton University Press.

Wheeler G.L., Brownlee C. (2008) Evolution of calcium channels in plants and green algae. Trends Plant Sci. 13: 506514.

Wildermuth M.C., Dewdney J., Wu G., Ausubel F.M. (2001) Isochorismate synthase is required to synthesize salicylic acid for plant defense. Nature 414: 562-565.

Wildon D.C., Thain J.F., Minchin P.E.H., Gubb I.R., Reilly A.J., Skipper Y.D., Doherty H.M., Odonnell P.J., Bowles D.J. (1992) Electrical signaling and systemic proteinaseinhibitor induction in the wounded plant. Nature 360: 6265.

Yamamoto H.Y., Bugos R.C., Hieber A.D. (1999) Biochemistry and molecular biology of the zanthophyll cycle. In: The Photochemistry of Carotenoids, ed. Frank H.A., Young A.J.K., Britton G., Cogdell R.J., Kluwer Academic Publishers, Dordrecht, p. 293-303.

Yasuda M., Ishikawa A., Jikumaru Y., Seki M., Umezawa T., Asami T., Maruyama-Nakashita A., Kudo T., Shinozaki K., Yoshida S., Nakashita H. (2008) Antagonistic interaction between systemic acquired resistance and the abscisic acid-mediated abiotic stress response in Arabidopsis. Plant Cell 20: 1678-1692. 